\title{
An oscillation criterion in $4 t h$-order neutral differential equations with a continuously distributed delay
}

\author{
George E. Chatzarakis ${ }^{1}$, Elmetwally M. Elabbasy ${ }^{2}$ and Omar Bazighifan ${ }^{3 *}$ (D)
}

"Correspondence:

o.bazighifan@gmail.com

${ }^{3}$ Department of Mathematics,

Faculty of Science, Hadhramout

University, Hadhramout, Yemen

Full list of author information is

available at the end of the article

\section{Springer}

\begin{abstract}
In this paper, a class of 4 th-order neutral delay differential equations with continuously distributed delay is studied. We establish a new oscillation criterion using the Riccati transformation. An example illustrating the results is also given.
\end{abstract}

MSC: $34 \mathrm{~K} 10 ; 34 \mathrm{~K} 11$

Keywords: 4th-order; Neutral differential equations; Oscillatory solutions

\section{Introduction}

In this work, we consider a 4 th-order neutral differential equation with a continuously distributed delay of the form

$$
\left[r(t)\left([x(t)+p(t) x(\tau(t))]^{\prime \prime \prime}\right)^{\alpha}\right]^{\prime}+\int_{a}^{b} q(t, \xi) f(x(g(t, \xi))) d \xi=0 .
$$

We assume that the following conditions hold:

$\left(H_{1}\right) \alpha$ is a quotient of odd positive integers;

$\left(H_{2}\right) p, q, \tau, g \in C\left(\left[t_{0}, \infty\right), R\right), r(t)$, and $q(t, \xi)$ are positive, $0 \leq p(t) \leq p<1, r(t) \in$ $C^{1}\left(\left[t_{0},+\infty\right)\right), r^{\prime}(t) \geq 0, \tau(t) \leq t, g(t, \xi) \leq t, \lim _{t \rightarrow \infty} \tau(t)=\infty, \lim _{t \rightarrow \infty} g(t, \xi)=$ $\infty, q(t, \xi)$ is not zero on any half line $\left[t_{\lambda}, \infty\right) \times[a, b], t_{\lambda} \geq t_{0}$, for $t \geq t_{0}, \xi \in[a, b]$, $g(t, \xi)$ is nondecreasing with respect to $\xi$.

$\left(H_{3}\right)$ There exists a constant $k>0$ such that $f(u) / u^{\gamma} \geq k$ for $u \neq 0$.

We define the corresponding function $z(t)$ of a solution $x(t)$ of (1) by $z(t)=x(t)+$ $p(t) x(\tau(t))$, we mean a non-trivial real function $x(t) \in C\left(\left[t_{x}, \infty\right)\right), t_{x} \geq t_{0}$, satisfying (1) on $\left[t_{x}, \infty\right)$ and, moreover, having the properties: $z(t), z^{\prime}(t), z^{\prime \prime}(t)$ and $r(t)\left[z^{\prime \prime \prime}(t)\right]^{\alpha}$ are continuously differentiable for all $t \in\left[t_{x}, \infty\right)$. We consider only those solutions $x(t)$ of (1) which satisfy $\sup \{|x(t)|: t \geq T\}>0$ for any $T \geq t_{x}$. A solution of (1) is called oscillatory if it has arbitrary large zeros, otherwise it is called nonoscillatory.

The oscillations of higher- and fourth-order differential equations have been studied by several authors, and several techniques have been proposed for obtaining oscillatory criteria for higher- and fourth-order differential equations. For treatments on this subject, we refer the reader to the texts $[2,5,16-18,21]$ and the articles $[1,3-15,19-26]$. In what

(C) The Author(s) 2019. This article is distributed under the terms of the Creative Commons Attribution 4.0 International License (http://creativecommons.org/licenses/by/4.0/), which permits unrestricted use, distribution, and reproduction in any medium, provided you give appropriate credit to the original author(s) and the source, provide a link to the Creative Commons license, and indicate if changes were made. 
follows, we review some results that have provided the background and motivation for the present work.

Cesarano and Bazighifan [8], Moaaz et al. [21], and Zhang et al. [26] studied the oscillation of the fourth-order nonlinear differential equation with a continuously distributed delay

$$
\left[r(t)\left(x^{\prime \prime \prime}(t)\right)^{\alpha}\right]^{\prime}+\int_{a}^{b} q(t, \xi) f(x(g(t, \xi))) d \xi=0 .
$$

Li et al. [19] studied the oscillatory behavior of the fourth-order nonlinear differential equation

$$
[r(t) z(t)]^{(4)}+q(t) x(\tau(t))=0
$$

Parhi and Tripathy [23] have considered the fourth-order neutral differential equations of the form

$$
\left[r(t)(y(t)+p(t) y(t-\tau))^{\prime \prime}\right]^{\prime \prime}+q(t) G(y(t-\sigma))=0
$$

and

$$
\left[r(t)(y(t)+p(t) y(t-\tau))^{\prime \prime}\right]^{\prime \prime}+q(t) G(y(t-\sigma))=f(t)
$$

and established the oscillation and asymptotic behavior of the above equations under the conditions

$$
\int_{t_{0}}^{\infty} \frac{1}{r^{\frac{1}{\alpha}}(t)} d \dot{t}<\infty
$$

and

$$
\int_{t_{0}}^{\infty} \frac{1}{r^{\frac{1}{\alpha}}(t)} d t=\infty
$$

respectively.

Our aim in the present paper is to use the Riccati method to establish new conditions for the oscillation of all solutions of (1) under condition (7).

The proof of our main results is essentially based on the following lemmas.

Lemma 1.1 Let $\beta \geq 1$ be a ratio of two numbers, where $U$ and $V$ are constants. Then

$$
U y-V y^{\frac{\beta+1}{\beta}} \leq \frac{\beta^{\beta}}{(\beta+1)^{\beta+1}} \frac{U^{\beta+1}}{V^{\beta}}, \quad V>0 .
$$

Lemma 1.2 If the function $z$ satisfies $z^{(i)}>0, i=0,1, \ldots, n$, and $z^{(n+1)}<0$, then

$$
\frac{z(t)}{t^{n} / n !} \geq \frac{z^{\prime}(t)}{t^{n-1} /(n-1) !}
$$


Lemma 1.3 Let $h \in C^{n}\left(\left[t_{0}, \infty\right),(0, \infty)\right)$. Assume that $h^{(n)}(t)$ is of a fixed sign and not identically zero on $\left[t_{0}, \infty\right)$ and that there exists $t_{1} \geq t_{0}$ such that $h^{(n-1)}(t) h^{(n)}(t) \leq 0$ for all $t \geq t_{1}$. If $\lim _{t \rightarrow \infty} h(t) \neq 0$, then for every $\lambda \in(0,1)$ there exists $t_{\lambda} \geq t_{0}$ such that

$$
h(t) \geq \frac{\lambda}{(n-1) !} t^{n-1}\left|h^{(n-1)}(t)\right| \quad \text { for all } t \geq t_{\lambda} .
$$

\section{Main results}

In this section, we shall establish some oscillation criteria for equation (1).

For convenience, we denote

$$
\begin{aligned}
& \eta(t)=\int_{t_{0}}^{\infty} r^{-1 / \alpha}(s) d s, \\
& \rho_{+}^{\prime}(t):=\max \left\{0, \rho^{\prime}(t)\right\}, \\
& \vartheta_{+}^{\prime}(t):=\max \left\{0, \vartheta^{\prime}(t)\right\}, \\
& Q(t)=\int_{a}^{b} q(t, \xi) d \xi
\end{aligned}
$$

and

$$
Q^{*}(v)=\int_{v}^{\infty} k Q(s)(1-p)^{\alpha}(g(s, a) / s)^{3 \alpha} d s
$$

Theorem 2.1 Assume that (7) holds. If there exist positive functions $\rho, \vartheta \in C\left(\left[t_{0}, \infty\right)\right)$ such that

$$
\int_{t_{0}}^{\infty}\left(\Psi(s)-\frac{2^{\alpha}}{(\alpha+1)^{\alpha+1}} \frac{r(s)\left(\rho^{\prime}(s)\right)^{\alpha+1}}{\mu^{\alpha} s^{2 \alpha} \rho^{\alpha}(s)}\right) d s=\infty
$$

for some $\mu \in(0,1)$, where

$$
\Psi(t)=k \rho(t) Q(t)(1-p)^{\alpha}(g(t, a) / t)^{3 \alpha}
$$

and either

$$
\int_{t_{0}}^{\infty} k Q(s)(1-p)^{\alpha}(g(s, a) / s)^{\alpha} d s=\infty
$$

or

$$
\int_{t_{0}}^{\infty}\left(Q^{*}(v)\right)^{\frac{1}{\alpha}} r^{\frac{-1}{\alpha}}(v) d v=\infty
$$

or

$$
\int_{t_{0}}^{\infty}\left[\vartheta(t) \int_{t}^{\infty}\left(Q^{*}(v)\right)^{\frac{1}{\alpha}} r^{\frac{-1}{\alpha}}(v) d v-\frac{\vartheta_{+}^{\prime 2}(t)}{4 \vartheta(t)}\right] d t=\infty
$$

then all solutions of (1) are oscillatory. 
Proof Let $x$ be a nonoscillatory solution of equation (1) defined in the interval $\left[t_{0}, \infty\right)$. Without loss of generality, we can assume that $x(t)$ is eventually positive. It follows from (1) that there are two possible cases for $t \geq t_{1}$, where $t_{1} \geq t_{0}$ is sufficiently large:

$\left(C_{1}\right) z^{\prime}(t)>0, z^{\prime \prime}(t)>0, z^{\prime \prime \prime}(t)>0,\left(r(t)\left(z^{\prime \prime \prime}(t)\right)^{\alpha}\right)<0$,
$\left(C_{2}\right) z^{\prime}(t)>0, z^{\prime \prime}(t)<0, z^{\prime \prime \prime}(t)>0,\left(r(t)\left(z^{\prime \prime \prime}(t)\right)^{\alpha}\right)<0$.

Assume that Case $\left(C_{1}\right)$ holds. Since $\tau(t) \leq t$ and $z^{\prime}(t)>0$, we get

$$
\begin{aligned}
z(t) & =x(t)+p(t) x(\tau(t)), \\
x(t) & =z(t)-p(t) x(\tau(t)) \\
& \geq z(t)-p(t) z(\tau(t)) \\
& =(1-p(t)) z(t) .
\end{aligned}
$$

From equation (1), we see that

$$
\left(r(t)\left(z^{\prime \prime \prime}(t)\right)^{\alpha}\right)^{\prime}=-\int_{a}^{b} q(t, \xi) f(x(g(t, \xi))) d \xi
$$

so that

$$
\begin{aligned}
\left(r(t)\left(z^{\prime \prime \prime}(t)\right)^{\alpha}\right)^{\prime} & =-\int_{a}^{b} k q(t, \xi) x^{\alpha}(g(t, \xi)) d \xi \\
& \leq-\int_{a}^{b} k q(t, \xi)(1-p(g(t, \xi)))^{\alpha} z^{\alpha}(g(t, \xi)) d \xi
\end{aligned}
$$

Since $g(t, \xi)$ is nondecreasing with respect to $\xi$ and $z^{\prime}(t)>0$, we have

$$
z(g(t, a)) \leq z(g(t, \xi)) \leq z(g(t, b))
$$

Thus

$$
\begin{aligned}
\left(r(t)\left(z^{\prime \prime}(t)\right)^{\alpha}\right)^{\prime} & \leq-(1-p)^{\alpha} \int_{a}^{b} k q(t, \xi) z^{\alpha}(g(t, \xi)) d \xi \\
& \leq-k(1-p)^{\alpha} z^{\alpha}(g(t, a)) \int_{a}^{b} q(t, \xi) d \xi \\
& =-k Q(t)(1-p)^{\alpha} z^{\alpha}(g(t, a)) .
\end{aligned}
$$

Now, we define a generalized Riccati substitution by

$$
\omega(t):=\rho(t) \frac{r(t)\left(z^{\prime \prime \prime}(t)\right)^{\alpha}}{z^{\alpha}(t)} .
$$

Then $\omega(t)>0$. Differentiating and using (19), we obtain

$$
\begin{aligned}
\omega^{\prime}(t) \leq & \frac{\rho^{\prime}(t)}{\rho(t)} \omega(t)-k \rho(t) Q(t)(1-p)^{\alpha} \frac{z^{\alpha}(g(t, a))}{z^{\alpha}(t)} \\
& -\alpha \rho(t) \frac{r(t)\left(z^{\prime \prime \prime}(t)\right)^{\alpha}}{z^{\alpha+1}(t)} z^{\prime}(t) .
\end{aligned}
$$


From Lemma 1.2, we have that $z(t) \geq \frac{t}{3} z^{\prime}(t)$, and hence

$$
\frac{z(g(t, a))}{z(t)} \geq \frac{g^{3}(t, a)}{t^{3}}
$$

Since $r^{\prime}(t)>0$ and $\left(r(t)\left(z^{\prime \prime \prime}(t)\right)^{\alpha}\right)^{\prime} \leq 0$, we get $z^{(4)}(t)<0$. It follows from Lemma 1.3 that

$$
z^{\prime}(t) \geq \frac{\mu}{2} t^{2} z^{\prime \prime \prime}(t)
$$

for all $\mu \in(0,1)$ and every sufficiently large $t$. Thus, by (24), (25), and (26), we get

$$
\begin{aligned}
\omega^{\prime}(t) \leq & \frac{\rho^{\prime}(t)}{\rho(t)} \omega(t)-k \rho(t) Q(t)(1-p)^{\alpha}\left(\frac{g(t, a)}{t}\right)^{3 \alpha} \\
& -\alpha \mu \frac{t^{2}}{2 r^{1 / \alpha}(t) \rho^{1 / \alpha}(t)} \omega^{\frac{\alpha+1}{\alpha}}(t) .
\end{aligned}
$$

Using Lemma 1.1 with $U=\frac{\rho^{\prime}(t)}{\rho(t)}, V=\frac{\alpha \mu t^{2}}{2 r^{1 / \alpha}(t) \rho^{1 / \alpha}(t)}$ and $y=\omega$, we get

$$
\omega^{\prime}(t) \leq-\Psi(t)+\frac{2^{\alpha}}{(\alpha+1)^{\alpha+1}} \frac{r(t)\left(\rho^{\prime}(t)\right)^{\alpha+1}}{\mu^{\alpha} t^{2 \alpha} \rho^{\alpha}(t)} .
$$

This implies that

$$
\int_{t_{1}}^{t}\left(\Psi(s)-r(s)\left(\rho^{\prime}(s) / \alpha+1\right)^{\alpha+1}\left(\frac{2}{\mu s^{2} \rho(s)}\right)^{\alpha}\right) d s \leq \omega\left(t_{1}\right)
$$

for some $\mu \in(0,1)$ which contradicts (12).

Assume that Case $\left(C_{2}\right)$ holds. Integrating (1) from $t_{1}$ to $t$, we obtain

$$
-r\left(t_{1}\right)\left(z^{\prime \prime \prime}\left(t_{1}\right)\right)^{\alpha} \leq-\int_{t_{1}}^{t} k Q(t) x^{\alpha}(g(s, \xi)) d s .
$$

From $z^{\prime}(t)>0, x(t) \geq(1-p(t)) z(t)$ and $g(s, \xi) \leq t$, it follows that

$$
\int_{t_{1}}^{t} k Q(s)(1-p)^{\alpha} z^{\alpha}(g(s, a)) d s \leq r\left(t_{1}\right)\left(z^{\prime \prime \prime}\left(t_{1}\right)\right)^{\alpha}
$$

From (25), we get

$$
\int_{t_{1}}^{t} k Q(s)(1-p)^{\alpha}(g(s, a) / s)^{3 \alpha} d s \leq r\left(t_{1}\right)\left(\frac{z^{\prime \prime \prime}\left(t_{1}\right)}{z\left(t_{1}\right)}\right)^{\alpha},
$$

which contradicts (12). Integrating (1) from $t$ to $\infty$, we obtain

$$
-r(t)\left(z^{\prime \prime \prime}(t)\right)^{\alpha} \leq-\int_{t}^{\infty} k Q(t) x^{\alpha}(g(s, \xi)) d s
$$

By virtue of $z^{\prime}(t)>0, x(t) \geq(1-p(t)) z(t), g(s, \xi) \leq t$, and (25), we obtain

$$
-\left(z^{\prime \prime \prime}(t)\right)+\frac{z(t)}{r(t)^{1 \backslash \alpha}}\left[\int_{t}^{\infty} k Q(s)(1-p)^{\alpha}(g(s, a) / s)^{3 \alpha} d s\right]^{1 / \alpha} \leq 0
$$


Integrating (33) from $t_{1}$ to $t$, we get

$$
\int_{t_{1}}^{t} \frac{1}{r^{1 / \alpha}(v)}\left(\int_{v}^{\infty} k Q(s)(1-p)^{\alpha}(g(s, a) / s)^{3 \alpha} d s\right)^{1 / \alpha} d v \leq-\frac{z^{\prime \prime}\left(t_{1}\right)}{z\left(t_{1}\right)}
$$

This yields

$$
\int_{t_{1}}^{t}\left(Q^{*}(v)\right)^{\frac{1}{\alpha}} r^{\frac{-1}{\alpha}}(v) d v \leq-\frac{z^{\prime \prime}\left(t_{1}\right)}{z\left(t_{1}\right)}
$$

which contradicts (15). Integrating (33) from $t$ to $\infty$, we get

$$
\int_{t}^{\infty}\left(Q^{*}(v)\right)^{\frac{1}{\alpha}} r^{\frac{-1}{\alpha}}(v) d v \leq-\frac{z^{\prime \prime}(t)}{z(t)}
$$

so that

$$
z^{\prime \prime}(t)+z(t) \int_{t}^{\infty}\left(Q^{*}(v)\right)^{\frac{1}{\alpha}} r^{\frac{-1}{\alpha}}(v) d v \leq 0 .
$$

Now, we define the Riccati substitution

$$
\psi(t):=\vartheta(t) \frac{z^{\prime}(t)}{z(t)}
$$

then $\psi(t)>0$ for $t \geq t_{1}$ and

$$
\psi^{\prime}(t):=\vartheta^{\prime}(t) \frac{z^{\prime}(t)}{z(t)}+\vartheta(t) \frac{z^{\prime \prime}(t) z(t)-\left(z^{\prime}(t)\right)^{2}}{z^{2}(t)}
$$

From (37) and (38), it follows that

$$
\psi^{\prime}(t) \leq-\vartheta(t) \int_{t}^{\infty}\left(Q^{*}(v)\right)^{\frac{1}{\alpha}} r^{\frac{-1}{\alpha}}(v) d v+\frac{\left(\vartheta^{\prime}(t)\right)_{+}}{\vartheta(t)} \psi(t)-\frac{1}{\vartheta(t)} \psi^{2}(t) .
$$

Hence, we have

$$
\psi^{\prime}(t) \leq-\vartheta(t) \int_{t}^{\infty}\left(Q^{*}(v)\right)^{\frac{1}{\alpha}} r^{\frac{-1}{\alpha}}(v) d v+\frac{\left(\left(\vartheta^{\prime}(t)\right)_{+}\right)^{2}}{4 \vartheta(t)} .
$$

Integrating (41) from $t_{1}$ to $s$, we get

$$
\int_{t_{1}}^{s}\left(\vartheta(t) \int_{t}^{\infty}\left(Q^{*}(v)\right)^{\frac{1}{\alpha}} r^{\frac{-1}{\alpha}}(v) d v+\frac{\left(\left(\vartheta^{\prime}(t)\right)_{+}\right)^{2}}{4 \vartheta(t)}\right) d t \leq \psi\left(t_{1}\right)
$$

for all large s, which contradicts (16).

The proof of the theorem is complete.

Let $\rho(t)=t^{3}$ and $\vartheta(t)=t$. As a consequence of Theorem 2.1, we obtain the following oscillation criterion. 
Corollary Assume that (7) holds and for some constant $\lambda_{0} \in(0,1)$

$$
\int_{t_{0}}^{\infty}\left(\Psi^{*}(t)-\left(\frac{3}{\alpha+1}\right)^{\alpha+1}\left(\frac{2}{\lambda_{0}}\right)^{\alpha} t^{2-3 \alpha} r(t)\right) d s=\infty
$$

where

$$
\Psi^{*}(t)=k t^{3} Q(t)(1-p)^{\alpha}(g(t, a) / t)^{3 \alpha}
$$

If either (14) or (15) is satisfied, or

$$
\int_{t_{0}}^{\infty}\left[t \int_{t}^{\infty}\left(Q^{*}(v)\right)^{\frac{1}{\alpha}} r^{\frac{-1}{\alpha}}(v) d v-\frac{1}{4 t}\right] d t=\infty
$$

then all solutions of (1) are oscillatory.

\section{Example}

In this section, we give the following example to illustrate our main results.

Example Consider the differential equation

$$
\left(\left[x(t)+\frac{1}{2} x\left(\frac{t}{3}\right)\right]^{\prime \prime \prime}\right)^{\prime}+\int_{0}^{1}\left(v / t^{4}\right) \xi x\left(\frac{t-\xi}{2}\right) d \xi=0
$$

where $v>0$ is a constant. Let

$$
\begin{aligned}
& \alpha=1, \quad r(t)=1, \quad p(t)=\frac{1}{2}, \quad \tau(t)=\frac{t}{3}, \\
& g(t, a)=\frac{t}{2}, \quad q(t, \xi)=\left(v / t^{4}\right) \xi, \quad f(x)=x,
\end{aligned}
$$

we get

$$
\begin{aligned}
\eta(s) & =\int_{t_{0}}^{\infty} d s=\infty, \\
Q(t) & =\int_{0}^{1} q(t, \xi) d \xi=\frac{v}{2 t^{4}} \\
Q^{*}(t) & =\int_{t_{0}}^{\infty} k Q(s)(1-p)^{\alpha}(g(s, a) / s)^{3 \alpha} d s \\
& =\int_{t_{0}}^{\infty} \frac{v}{32 s^{4}} d s=\frac{v}{96 t^{3}} .
\end{aligned}
$$

If we now set $k=1$, then

$$
\begin{aligned}
& \int_{t_{0}}^{\infty}\left(k t^{3} Q(t)(1-p)^{\alpha}(g(t, a) / t)^{3 \alpha}-\left(\frac{3}{\alpha+1}\right)^{\alpha+1}\left(\frac{2}{\lambda_{0}}\right)^{\alpha} t^{2-3 \alpha} r(t)\right) d s \\
& \quad=\left(\frac{v}{32}-\frac{9}{2 \lambda_{0}}\right) \int_{t_{0}}^{\infty} \frac{1}{t} d t=\infty, \quad \text { if } v>\frac{144}{\lambda_{0}} \text { for some constant } \lambda_{0} \in(0,1),
\end{aligned}
$$


and

$$
\begin{aligned}
& \int_{t_{0}}^{\infty} {\left[t \int_{t}^{\infty}\left(Q^{*}(v)\right)^{\frac{1}{\alpha}} r^{\frac{-1}{\alpha}}(v) d v-\frac{1}{4 t}\right] d t } \\
& \quad=\int_{t_{0}}^{\infty}\left[t \int_{t}^{\infty} \frac{v}{96 t^{3}} d t-\frac{1}{4 t}\right] d t \\
& \quad=\int_{t_{0}}^{\infty}\left[t \frac{v}{192 t^{2}}-\frac{1}{4 t}\right] d t \\
& \quad=\left(\frac{v}{192}-\frac{1}{4}\right) \int_{t_{0}}^{\infty} \frac{1}{t} d t \\
& \quad=\infty, \quad \text { if } v>48 .
\end{aligned}
$$

Thus, by Corollary, every solution of equation (46) is oscillatory.

\section{Conclusion}

The results of this paper are presented in a form which is essentially new and of high degree of generality. In this paper, using a Riccati transformation technique, we offer some new sufficient conditions which ensure that any solution of Eq. (1) oscillates under the condition $\int_{t_{0}}^{\infty} \frac{1}{r^{\frac{1}{\alpha}}(t)} d t=\infty$. Further, we can consider the case of $z(t)=x(t)-p(t) x(\tau(t))$, and we can try to get some oscillation criteria of Eq. (1) in the future work.

\section{Acknowledgements}

The authors express their debt of gratitude to the editors and the anonymous referee for accurate reading of the manuscript and beneficial comments.

\section{Funding}

The authors received no direct funding for this work.

\section{Competing interests}

The authors declare that there are no competing interests between them.

\section{Authors' contributions}

The authors declare that they have read and approved the final manuscript.

\section{Author details}

${ }^{1}$ Department of Electrical and Electronic Engineering Educators, School of Pedagogical and Technological Education (ASPETE), Athens, Greece. ${ }^{2}$ Department of Mathematics, Faculty of Science, Mansoura University, Mansoura, Egypt.

${ }^{3}$ Department of Mathematics, Faculty of Science, Hadhramout University, Hadhramout, Yemen.

\section{Publisher's Note}

Springer Nature remains neutral with regard to jurisdictional claims in published maps and institutional affiliations.

Received: 17 January 2019 Accepted: 1 August 2019 Published online: 09 August 2019

\section{References}

1. Agarwal, R., Grace, S., Manojlovic, J.: Oscillation criteria for certain fourth order nonlinear functional differential equations. Math. Comput. Model. 44, 163-187 (2006)

2. Agarwal, R., Grace, S., O'Regan, D.: Oscillation Theory for Difference and Functional Differential Equations. Kluwer Academic, Dordrecht (2000)

3. Agarwal, R., Grace, S., O'Regan, D.: Oscillation criteria for certain nth order differential equations with deviating arguments. J. Math. Anal. Appl. 262, 601-622 (2001)

4. Baculikova, B., Dzurina, J., Graef, J.R.: On the oscillation of higher-order delay differential equations. Math. Slovaca 187, 387-400 (2012)

5. Bazighifan, O.: Oscillatory behavior of higher-order delay differential equations. Gen. Lett. Math. 2, 105-110 (2017)

6. Bazighifan, O., Cesarano, C.: Some new oscillation criteria for second-order neutral differential equations with delayed arguments. Mathematics 7, 1-8 (2019)

7. Bazighifan, O., Elabbasy, E.M., Moaaz, O.: Oscillation of higher-order differential equations with distributed delay. J. Inequal. Appl. 2019, 55 (2019) 
8. Cesarano, C., Bazighifan, O.: Oscillation of fourth-order functional differential equations with distributed delay. Axioms 7, 1-9 (2019)

9. Cesarano, C., Bazighifan, O.: Qualitative behavior of solutions of second order differential equations. Symmetry 11, $1-8(2019)$

10. Cesarano, C., Pinelas, S., Al-Showaikh, F., Bazighifan, O.: Asymptotic properties of solutions of fourth-order delay differential equations. Symmetry 11, 1-10 (2019)

11. Elabbasy, E.M., Hassan, T.S., Moaaz, O.: Oscillation behavior of second order nonlinear neutral differential equations with deviating arguments. Opuscula Mathematica 32, 719-730 (2012)

12. Grace, S., Graef, J., Tunc, E.: Oscillatory behavior of a third order neutral dynamic equations with distributed delays. Electron. J. Qual. Theory Differ. Equ. 2016, 14 (2016)

13. Grace, S., Graef, J., Tunc, E.: Oscillatory behavior of second order damped neutral differential equations with distributed deviating arguments. Miskolc Math. Notes 18, 759-769 (2017)

14. Grace, S., Lalli, B.: Oscillation theorems for nth order nonlinear differential equations with deviating arguments. Proc. Am. Math. Soc. 90, 65-70 (1984)

15. Graef, J., Tunc, E.: Oscillation of fourth-order nonlinear dynamic equations on time scales. Panam. Math. J. 25, 16-34 (2015)

16. Gyori, I., Ladas, G.: Oscillation Theory of Delay Differential Equations with Applications. Clarendon, Oxford (1991)

17. Kiguradze, I., Chanturia, T.: Asymptotic Properties of Solutions of Nonautonomous Ordinary Differential Equations. Kluwer Academic, Drodrcht (1993)

18. Ladde, G., Lakshmikantham, V., Zhang, B.: Oscillation Theory of Differential Equations with Deviating Arguments. Dekker, New York (1987)

19. Li, T., Baculikova, B., Dzurina, J., Zhang, C.: Oscillation of fourth order neutral differential equations with p-Laplacian like operators. Bound. Value Probl. 2014, 56 (2014)

20. Moaaz, O., Chalishajar, D., Bazighifan, O.: Some qualitative behavior of solutions of general class difference equations. Mathematics 7, 1-12 (2019)

21. Moaaz, O., Elabbasy, E.M., Bazighifan, O.: On the asymptotic behavior of fourth-order functional differential equations. Adv. Differ. Equ. 2017, 261 (2017)

22. Moaaz, O., Elabbasy, E.M., Muhib, A.: Oscillation criteria for even-order neutral differential equations with distributed deviating arguments. Adv. Differ. Equ. 2019, 297 (2019)

23. Parhi, N., Tripathy, A.: On oscillatory fourth order linear neutral differential equations-I. Math. Slovaca 54, 389-410 (2004)

24. Philos, C.: On the existence of nonoscillatory solutions tending to zero at $\infty$ for differential equations with positive delay. Arch. Math. (Basel) 36, 168-178 (1981)

25. Tunc, E., Graef, J.: Oscillation results for second order neutral dynamic equations with distributed deviating arguments. Dyn. Syst. Appl. 3, 289-303 (2014)

26. Zhang, C., Li, T., Saker, S.: Oscillation of fourth-order delay differential equations. J. Math. Sci. 201, 296-308 (2014)

\section{Submit your manuscript to a SpringerOpen ${ }^{\circ}$ journal and benefit from:}

- Convenient online submission

- Rigorous peer review

- Open access: articles freely available online

- High visibility within the field

- Retaining the copyright to your article

Submit your next manuscript at $\gg$ springeropen.com 